\title{
Perspectives of patients towards medical students at the Queen Elizabeth Central Hospital, Blantyre, Malawi
}

\author{
M. MOLAPO and A.S. MUULA* \\ Department of Public Health, Division of Community Health, University of Malawi, College of Medicine, \\ Private Bag 360, Chichiri, Blantyre 3, Malawi
}

\begin{abstract}
Medical students gaining experience in outpatient's clinics and admission wards are exposed to clinical clerkship as this is an important part of their training. There is paucity of reported patients' experiences and perceptions of medical students in low-income settings. The present study was conducted at the Queen Elizabeth Central Hospital $(\mathrm{QECH})$, Malawi to describe patients' and perceptions of, and experience with medical students being present when patients are seen by a medical doctor or students' individual clerking. Participants mostly felt that medical students were compassionate, enthusiastic and helpful to them. The participants' who had been ever clerked by students felt motivated to be present for student learning for altruistic reasons as well as for mutual benefit between student and patient. Patients attending the QECH in Blantyre, Malawi are generally satisfied with the attention and support they obtain from students. Balancing the need for appropriate supervision of students, and facilitating the retention of this patient-cantered approach to care should be maintained.
\end{abstract}

Keywords: medical training, medical students, medical ethics, Malawi,

\section{Introduction}

Clinical clerkship, whether in outpatients' clinics or admission wards, is an important aspect in the training of medical students. These settings and experiences provide the opportunity for medical students to learn clinical skills through observation and practice. Different medical schools differ in the time they introduce this important experience in the medical curriculum.

The tendency, however, is such that many medical schools are introducing clinical exposure of students early in the curriculum (McLean, 2004). Whether students are introduced early to patient contact or later in the curriculum, each option is likely to have its own benefits and challenges. An early start may reinforce the students' interest in medicine, as this may provide opportunity to immediately link basic sciences and clinical medicine; early exposure to patient contact can facilitate the integration of basic sciences and clinical skills. However, students may not have sufficient background knowledge in basic sciences to enable them make the best out of clinical exposure.

The history of Malawi's only medical school has been described elsewhere (Broadhead, 1998; Dahlenburg, 1993). In Malawi, structured clinical rotations, of between 7 and 9 weeks, do not start until students are in their third year of medical school. Since its inception in 1991 to 2000, the number of students per year of study has not exceeded 25 . Recently however, there has been an increase in the annual student intake to between 40 and 60 (Muula, 2005), necessitating the need for another teaching hospital. The curriculum has also gone through several rounds of revisions culminating in the decision to introduce students to clinical environments from the first year. The increase in student enrolment has

\footnotetext{
* Correspondence: Dr. Adamson S. Muula; E-mail: amuula@medcol.mw
} 
been facilitated by the establishment of a premedical course at the College which is serving as a source for candidates, increased numbers of teaching staff as a result of former graduates returning from postgraduate training and committed funding from government and the Global Fund Against HIV/AIDS, Tuberculosis and Malaria (The Global Fund).

Supervised clinical interaction between medical students and patients is an important resource for the training of future doctors. Patients' perceptions towards the presence of medical students during their medical consultations may influence their behaviour in such a way that the quality of learning that the student gets may be affected. We are not aware of any previous studies in Malawi which have documented patients' perceptions and their assessment of students in clinical settings. We believe this information is useful in modifying the orientation that students have before entering the clinical setting. The aims of the research were to describe patients' experiences with medical students and to identify patients' concerns with students being present during clinical consultations.

\section{Materials and Methods}

\section{Study design}

Due to the exploratory nature of the research topic, cross sectional qualitative research methods using focus group discussions (FGDs) and content analysis were considered appropriate (Trauth, 2001). Limited resources allowed only four FGDs to be conducted.

Four FGDs were held at the Queen Elizabeth Central Hospital (QECH), Blantyre, Malawi in 2006. Study participants sampling used a non-probability convenience sample of hospital attendees from antenatal ward, adult general female and general male wards and family planning clinic. The wards sampled were chosen so as to recruit both male and female patients, as well as women attending for maternity care. Paediatrics and surgical patients were not included.

One of the authors (MM) and a research assistant recruited volunteer patients from the wards after the study had been explained to them. The inclusion criteria were that these should be patients within the selected wards, conscious and having expressed willingness to participate in the study. Patients who were unable to provide consent were excluded from the study.

The FGDs conducted in Chichewa (the local language and Malawi's one of the official languages) took about an hour and were taped. Data was then transcribed before translation into English. A study guide was used, and further exploratory questions were asked as the discussions were allowed to take a natural flow. Following the FGDs, participants were provided with refreshments (sodas and scones). Each of the FGDs comprised members of the same sex. Analysis of the transcripts was done manually based on themes (i.e., comparisons of patients' experiences with doctors compared to medical students; assessment of patient-student interactions; and ethical concerns) were identified. Particularly informative statements were also isolated. The research protocol was reviewed and approved by the Malawi College of Medicine Research and Ethics Committee.

\section{Data analysis}

Data were analysed by content analysis (Reissman, 1993; Denzin, 1997; Whitley, 2004).The transcripts were used to generate coded keywords and phrases. These were re-examined to confirm or modify the original keywords but also to generate new codes (de wet \& Erasmus, 2005; Whitley, 2004). This was done in order to identify recurring themes, compare among 
the different groups and identify outliers. The results of this analysis and our interpretations are presented below.

\section{Results}

\section{Characteristics of the study participants}

Four focus group discussions were held with a total of 29 participants who were recruited from the family planning clinic, adult general medical wards (both male and female) and the antenatal ward. The antenatal ward comprises women with high risk pregnancies, but not yet in labour. Age ranged from 15-74 years. Twenty-four participants were females where only 5 were males; 18 were married, 10 single and 1 widowed. Seventeen participants had attained various levels of primary education, 10 secondary education and 2 did not wish to specify. The mean highest school grade attained for family planning was 7, standard 6 for male patients, 8 for female medical patients and 10 for antenatal ward patients.

\section{Differences between a doctor and a medical student}

As there was concern that perhaps in a less educated community as Malawi, patients may not be able to distinguish between medical students and doctors, FGD participants were specifically asked how they may be able to distinguish a student from a doctor. Study participants indicated they were able to know who a medical student was through a diversity of means like the nature of care they gave patients, their demeanour, enthusiasm and lack of confidence but also attention to detail. As can be determined from a participant's response, the times that a student was present in the ward were suggestive to some patients:

'I think the main difference between a doctor and a student is that the doctor comes frequently, while a student has a specific time at which they come. Most of the patients don't know what the difference is between a doctor or a nurse and a student. They just think that all are doctors.'

While both perceptions, i.e., that student could be identified easily but also that some patients may not be able to distinguish between a doctor and patient, another participant indicated:

'We can't differentiate. They themselves know who is a doctor and who is a student.'

Students are required to wear an identification badge. However, there are concerns that in a predominantly less literate society, name tags may be less meaningful than logos. A male participant said:

'Let me comment a bit. A person who is a bit educated, when they see the logo of the University of Malawi, they can tell the person with the identity card is a student, not yet a doctor.'

It is worthy to note that participants described a doctor as older and a student as younger but also used racial terms to distinguish a student from a doctor. A participant remarked:

'Just as we said, we just see a lot of people in white coats. But when there is a white man among them, we do know that the white man is the doctor and the rest are students.'

Participants also noted that the lack of authority was indicative of who was in charge and who was a student at clinics. A woman participant at the family planning clinic said:

'When we came here this morning, we knew these are students and those are the ones who run the clinic, because when students come here, they wait until the nurse comes and lead them.' 


\section{Medical students are compassionate}

In all the FGDs, participants showed reasonable satisfaction with the way students had interacted with the patients, especially in so far as the students participated in the care and support of the patient. A participant said:

'When we explain our problems to the students, they take time to listen, unlike doctors who do not listen but just go on prescribing medications without listening.'

A women medical patient indicated that students were not perceived as always helpful.

'Sometimes when students are on duty, they do not care about patients. This has happened to me before. I brought my son to hospital. He had a wound on the head and I was told by the students that I should just clean the wound with salty-water. I later booked an appointment to see a white doctor whom I know would assist me. My son was admitted and the wound cleaned and later on it healed. At times students do not care at all.'

\section{Lack of communication between care team and patient}

The FGDs also highlighted that there was sometimes lack of communication between patients and the care teams. Patients were not always approving of the discussions that occur during clinic times or ward rounds amongst students and their clinical supervisors. This was much so when it was perceived that patients' concerns were not addressed to the patients' satisfaction. An illustrative example came from a participant who said:

'When we came here, there was a woman who attended us, but then another patient came and without having finished with us, she went on to attend to that patient. Health professionals are busy having discussions among themselves, instead of concentrating on the patient. I witnessed this yesterday.'

Another participant said:

'There is lack of concentration when you people are discussing. You then come back to ask; 'What is your name again?' Yet you had already asked that question.'

\section{Respect for patient autonomy}

We also aimed to assess whether in most situations where the patients has had students present during clinical or ward consultation, their consent was explicitly sought. In general, this was not the case, although there were exceptions. A participant reporting about clinic consultations said:

'When a patient comes into a consultation room, and he finds a number of people who are wearing white coats, it is not possible for him to choose who should remain in the room when he is being seen.'

It is likely that in these situations, even though the patient has considered that they may have the right to request not to have students around, they felt helpless to ask for that. Another participant suggested that possibly patients coming to a government (public) hospital where patients are not required to pay anything, their wishes could not be respected far enough. A participant said:

'It is very difficult to choose who has to see you in a government hospital. You just accept anyone who comes along. I don't think there is such a thing as being given an opportunity to make a choice.'

The experience of not having been asked for permission to have students around was not universal as can be understood in the following response from one of the participants: 
'It happened to my mother when she came to deliver the last born in our family. She was assisted by students.... She was told that students would be present and she accepted that they should be there.'

Participants in the antenatal ward also reported that oftentimes where patients were being sought for demonstrations, they (the patients) would be given opportunity to volunteer or not if so they wished.

\section{What do students do with patients' information?}

We had thought that the way patients perceived students and responded to students-related requests was likely to be influenced by what the patients thought the information the students collected was used. A participant reported:

'There was a time I was examined by a student who came later to see me with a doctor and the student gave a report to the doctor.'

Many participants also indicated that students were crucial to their care through advocating on their behalf and acting as liaison between the patient and staff, engaging in patient education and in some cases, providing direct care. While for most participants, they perceived students as being professional and that they would keep confidentiality, there were still concerns about confidentiality. A participant said:

'I know that somehow they cannot always keep secrets. They may be telling their friends. But even if they did that, they will still not know the person with the condition. If patients worry that their secrets will be revealed, they may not come to hospital after all.'

Perhaps not surprising, an antenatal ward respondent said:

'It is in the labour ward that one gets really embarrassed because you ask yourself, 'Should these kids see me naked?'

Another woman gave her own previous experience:

'I do get worried about such matters. After I delivered my second-born, I happened to overhear some students discussing amongst themselves the things that I was doing in labour, such as crying. My niece was one of the students and I confronted her afterwards to ask her why she had watched me during labour, only to discuss later with her friends.'

It was interesting to note that the discussions that students and their clinical teachers have about the patient in their presence could be a matter of concern to the patient. A participant talking about these discussions said:

'It is good if they sit in their office and discuss amongst themselves in a confined place. They should not be discussing the patients when she is around because she may feel uncomfortable and that is not being polite.'

Patients also reported that students were 'too busy' to spend time talking about a particular patients' history outside the care setting. Some also observed that medical students saw a lot of patients, many of whom not personally known to them (the student or their friends) that talking about a particular patient in a way that the patient's identity would be known was remote. An illustrative situation was when a participant said:

'I would say that when a patient has HIV and the patient and the doctor knows, the patient is not worried that they will tell his or her secret to other people because the patient has never met the doctor before.'

\section{Patients' motivations in being available}

It is important to identify what are some of the motivations of patients or what the patients perceive as to their role in the teaching of medical students enterprise. Participants indicated 
altruistic motives, indicated a sense of mutualism between the patient and the medical student. A participant said:

'We are happy that we are contributing to the training of doctors.'

Another participant said:

'I am glad because we help each other, as a patient I help the student so that the student can help me and other people.'

This sense of satisfaction with the patient-student interaction was also accompanied by a sense of betrayal:

'This college is a government institution, yet after students train, they go to work in other hospitals. May be you do that because the government gives doctors low wages.'

It is worth noting that while participants felt the public health sector should not be abandoned after the medical students have trained, they also recognized that life may be tough financially for the qualified doctor within the public health sector.

\section{Discussion}

In general, this qualitative study showed that even in a predominantly less educated society, patients were able to distinguish between a medical student and a doctor. Medical students were identified as usually young, enthusiastic in their undertakings with patients, detailed in the medical interview, showing less authority but demonstrating empathy. Patients' observations and assessments however were not validated, and therefore it is not known if their assessments were accurate or not. However, the fact that patients perceived students as compassionate and useful may facilitate better patient-student interaction.

Participants expressed concerns on lack of respect for patient autonomy in that in general patients were not requested whether they approve of the presence of medical students during clinical consultations, concerns about confidentiality, and compromised care.

The reports that staff sometimes mistreats patients is of particular concern; there were reports that women in labour had been described as 'loud'. Shapiro et al (2006) reported that medical students in the United States had observed that 'Some residents talk about patients doing the Macarena, which means, Latina women in labour are loud.' In such a setting, this implied that medical students had been exposed to negative attitudes towards patients by their seniors.

Fortier et al. (2006) in their study of 367 patients reported that compared to younger patients, older patients were less willing to be seen by a medical student. In our study, some women reported unwillingness to be seen in the delivery room by a young medical student. However, this was not universal as some participants reported positive experiences after being seen by students.

Gender may affect the willingness by student to be seen by students. Patients' unwillingness to be seen by a particular gender of medical students and the reported unwillingness of women in particular to be seen by male students however is not universal. Shann \& Wilson (2006) reported on a patients' acceptability study on being examined by medical students in the UK. In general younger women and men, those visiting the clinic for the first time, and women with no children were less likely to accept a student of either sex to take their history or observe their examination.

Monnickendam et al (2001) in a study from Israel reported that only 3.2\% of patients objected to having students during clinic consultation and $4 \%$ thought that presence of 
students was detrimental to history taking and physical examination. It was reported by $15 \%$ that they would insist on advance notification.

Patients attending a teaching hospital should be aware that they may be asked to be seen by a student. In the case of the QECH however, the patient's very life may be dependent on the student especially due to the shortage of qualified health professionals. Participants in this study displayed realistic attitudes as students may take up patient care roles that they would not normally take in other medical schools. The perception, however, that because QECH is a government health facility then patients do not have some choice ought to be discouraged. This may however be inadvertently displayed or in fact is the perception held by students and medical teachers as patients in paying wards are less likely to be recruited for patient teaching compared to their non-paying counterparts.

The respect for patient autonomy and right to not participate in student learning must be balanced with patients' citizenship responsibilities and reciprocity (Maa \& McCullough, 2006). If all patients refused to take part in student teaching, medical education would change considerably. The patient of today must realize that $\mathrm{s} /$ he is being seen by a doctor because past patients participated in student teaching.

It was particularly interesting to note that even in this setting where HIV and AIDS discussion are likely to feature heavily in dealing with patients, participants while noting the sensitivity of the disease, did not unusually express an overt need to extra confidentiality and privacy. In a study of nursing students, Sharif \& Masoumi (2005) suggested that differences between actual and expected behaviour in the clinical placement of nursestudents, creates conflicts. In our situation, it is a matter of interest as to how medical students resolve, if at all, the conflicts they have to deal with during clinical placement when they learn that what they are taught is not necessarily how qualified doctors practice.

The low socio-economic, including low education status and lack of viable options for health care among the study participants may have been among the reasons study participants accepted the presence of medical students. There are almost always concerns about how representative the findings from a qualitative study can be to the source population. These concerns are mostly usually misplaced as qualitative studies are generally not meant to produce generalisable results but afford an in-depth understanding of an issue or issues. However, as the responses to the questions were generally similar from one FGD to the other suggested that the reports were a reasonable reflection of the perception of the patient population at QECH, Malawi.

In a study like this, there are bound to be different opinions expressed by study participants. We noted that while most participants reported positive experiences with medical students, others did not. Although participants had perceptions on medical students and doctors, some had also indicated that they may not be able to differentiate as to who was a doctor and who was a medical student. If participants had used race, age and demeanour alone to distinguish a doctor and a medical student, it is possible that some of the attributes of one group (e.g. medical students or doctors) may have been ascribed to the other. That patient can mistakenly consider a medical student as if they were a qualified doctor has also been described by Maa \& McCullough (2006). However, from the FGDs, we believe that while patients can and do err in distinguishing a medical student from a doctor, most of their identification marks were reasonable.

Lastly, this study had a number of limitations. This study used convenience sampling to recruit participants, thus limiting its generalizability to the whole patient population at QECH or other teaching hospitals. Secondly, cultural nuances and messages 
are often communicated via body language and other non-verbal cues. These were not analysed in the present study. Finally, interpretation of interviewee comments as being literally true and accurate may be problematic as these may be subject to bias that can be introduced through the individuals' concerns of social desirability (May \& Fixcroft, 1995).

In conclusion, we suggest that there be a concerted effort by the Malawi College of Medicine and the Queen Elizabeth Central Hospital to raise patient and community awareness on the role of a teaching health facility. With the input of the community and other relevant stakeholders, there should be a way found on how to remind patients of their rights and responsibilities. The intention should not be to restrict patients' participation in clinical teaching, but rather to facilitate such instruction which should only occur when both parties involves are aware of what is going on. Finally, as patients obtain significant benefits from being "managed" by students, it is also important that any harm that may be realized as a result of student involvement in patient care is minimized.

The interaction between medical students and patients in Malawi appear to be patient-cantered aimed at not only facilitate learning, but also provide care and support to patients. Medical students are perceived by patients as enthusiastic and detailed in their undertaking. There is need to nurture the mutual relationship between the student and the patient.

\section{Competing interests}

The authors declare no competing interests.

\section{Acknowledgements}

We are grateful to Owen Henry and Gift Mulima who assisted in data collection and Vincent Jumbe and Paul Ndebele for logistical support and assistance with data analysis. Funding from this research was provided by University of Malawi-College of Medicine Student Research Grant.

Received 31 August 2009

Revised 9 December 2009

Accepted 10 December 2009

\section{References}

Broadhead, R.L. (1998) Community-based paediatric curriculum: the Malawi experience. Annals of Tropical Paediatrics 18 Suppl, S27-s32.

Dahlenburg, G.W. (2003) A letter from-Malawi: the first year of the College of Medicine of the University of Malawi. Tropical Doctor 23, 4-6.

Denzin, N.K. (1997) Interpretive Ethnography: Ethnographic Practices for the 21 $1^{\text {st }}$ Century. Thousand Oakes, California, Sage.

de Wet, J. \& Erasmus, Z. (2005) Riguor in qualitative research: tracing the debate. Qualitative Research Journal 5, 28-40.

Fortier, A.M., Hahn, P.M., Trueman, J. \& Reid, R.L. (2006) The acceptance of medical students by women with gynaecology appointments. Journal of Obstetric and Gynaecology Canada 28, 526-530. 
Maa, A. \& McCullough, L.B. (2006) Medical education in the public versus the private setting: a qualitative study of medical students' attitudes. Medical Teacher 28, 351-55.

May, C. \& Foxcroft, D. (1995) Minimizing bias in self reports of health beliefs and behaviors. Health Education Research 10, 107-112.

McManus, I.C., Keeling, A. \& Paice, E. (2004) Stress, burn out and doctors' attitudes to work are determined by personality and learning styles: A twelve year longitudinal study of UK medical graduates. BMC Medicine 2, 29.

McLean, M. (2004) Sometimes we do it right. Early clinical contact is a rewarding experience. Education for Health (Abingdon) 17, 42-52.

Monnickendam, S.M., Vinker, S., Zalewski, S., Cohen, O., Kitai, F. (2001) Patients' attitudes towards the presence of medical students in family practice consultations. Israel Medical Association Journal 3, 903-906.

Muula, A.S. (2005) Five-year experience of Malawi College of Medicine with 'learning by living' program. Croatian Medical Journal 46, 1010-1014.

Reissman, C.K. (1993) Narrative analysis. Newbury Park, California, Sage Publications.

Shann, S., Wilson, J.D. (2006) Patients' attitudes to the presence of medical students in a genitourinary medical clinic: a cross sectional survey. Sexually Transmitted Infections $82,52-54$.

Shapiro, J., Lie, D., Gutierrez, D. \& Zhuang, G. (2006) 'That never would have occurred to me'. A qualitative study of medical students' views of a cultural competence curriculum. BMC Medical Education 6, 31.

Sharif, F. \& Masoumi, S. (2005) A qualitative study of nursing students experiences of clinical practice. BMC Nursing 4, 6 .

Trauth, E.M. (2001) The Choice of Qualitative Methods in Issues Research. Idea Group Publishing, Hershey, Pennsylvania, United States, p27-32.

Whitley, A. (2004). Grounded research: a modified grounded theory for the business setting. Qualitative Research Journal 2, 27-42. 